\title{
A NOTE ON THE GENUS OF GLOBAL FUNCTION FIELDS
}

\author{
ZHENGJUN ZHAO \\ School of Mathematics and Computational Science, AnQing Normal \\ University, AnQing 246133, People's Republic of China \\ e-mail: zywnju@gmail.com \\ and XIA WU* \\ Department of Mathematics, Southeast University, \\ Nanjing 210096, People's Republic of China \\ e-mail:xiaxia80@gmail.com
}

(Received 19 March 2012; revised 5 August 2012; accepted 8 November 2012; first published online 26 February 2013)

\begin{abstract}
To give a relatively elementary proof of the Brumer-Stark conjecture in a function field context involving no algebraic geometry beyond the RiemannRoch theorem for curves, Hayes (Compos. Math., vol. 55, 1985, pp. 209-239) defined a normalizing field $H_{\mathfrak{e}}^{*}$ associated with a fixed sgn-normalized Drinfeld module and its extension field $K_{\mathfrak{m}}$, which is an analogue of cyclotomic function fields over a rational function field. We present explicitly in this note the formulae for the genus of the two fields and the maximal real subfield $H_{\mathfrak{m}}$ of $K_{\mathfrak{m}}$. In some sense, our results can be regarded as generalizations of formulae for the genus of classical cyclotomic function fields obtained by Hayes (Trans. Amer. Math. Soc., vol. 189, 1974, pp. 77-91) and Kida and Murabayashi (Tokyo J. Math., vol. 14(1), 1991, pp. 45-56).
\end{abstract}

2010 Mathematics Subject Classification. 11R58, 11R60, 11G09.

1. Introduction. Let $g_{L}$ be the genus of the algebraic function field $L / F$ with a constant field $F$. The genus is important and intrinsic to any function field. It is well known that it is a difficult task to compute the genus of the function field in many cases. Perhaps the most powerful tool to deal with it is the Riemann-Hurwitz formula, which relates the genus $g_{L}$ with the genus $g_{K}$ of a subfield $F \subseteq K \subseteq L$ of finite degree $[L: K]<\infty$. The Riemann-Hurwitz formula tells us that the key point to compute the genus is by determining the different divisor $D_{L / K}$ of the extension $L / K$, which is a divisor of $L$ and contains all prime divisors of $L$ that are ramified in $L / K$.

To provide an explicit class field theory for the rational function field, Hayes investigated carefully in 1973 the cyclotomic function fields which are analogues of cyclotomic number fields. Let $k=\mathbb{F}_{q}(T)$ be a rational function field over a finite field $\mathbb{F}_{q}$ with $q$ elements. Denote by $\infty$ the infinite prime divisor $\frac{1}{T}$ of $k$. Let $\Lambda_{M}$ be a set of $M$-torsion points of $k^{a c}$ associated with the Carlitz module, where $M$ is a polynomial of positive degree in $\mathbb{F}_{q}[T]$ and $k^{a c}$ is the algebraic closure of $k . K_{M}=k\left(\Lambda_{M}\right)$ are called cyclotomic function fields in [6]. Let $G_{\infty}$ be the decomposition group of $\infty$ in $K_{M} / k$ for $M \in \mathbb{F}_{q}[T]$ of positive degree. The fixed field of $G_{\infty}$ in $K_{M}$, denoted by $K_{M}^{+}$, is called the maximal real subfield of $K_{M}$. It is well known ([6], chapter 12) that $K_{M} / k$ is an

${ }^{*}$ Corresponding author. 
abelian extension and the properties of $K_{M}^{+}$are similar to those of the maximal real subfield of a cyclotomic number field.

Let $P$ be a monic irreducible polynomial of degree $d>0$ in $\mathbb{F}_{q}[T]$. Hayes gave in [1] the explicit formula for the genus of $K_{P^{n}}$, where $n$ is a positive integer.

THEOREM 1.1 ([1], Corollary 4.2). Let $\Phi(M)$ denote the order of the multiplicative group $\left(\mathbb{F}_{q}[T] /(M)\right)^{*}$ for a polynomial $M \in \mathbb{F}_{q}[T]$ with positive degree. Then

$$
2 g_{K_{P^{n}}}-2=(d q n-d n-q) \frac{\Phi\left(P^{n}\right)}{q-1}-d q^{d(n-1)} .
$$

Based on Hayes's ideas in [1], Kida and Murabayashi [5] calculated the genus for $K_{M}$ and $K_{M}^{+}$with respect to the arbitrary polynomial $M \in \mathbb{F}_{q}[T]$ of positive degree.

THEOREM 1.2 ([5], Corollary 1). Let $M=\prod_{i=1}^{r} P_{i}^{n_{i}}$ be the factorization of $M \in$ $\mathbb{F}_{q}[T]$ into powers of monic irreducible polynomials $P_{i}$ and $d_{i}=\operatorname{deg}\left(P_{i}\right), i=1, \ldots, r$. Let $s_{i}=n_{i} \Phi\left(P_{i}^{n_{i}}\right)-q^{d_{i}\left(n_{i}-1\right)}$. Then

$$
2 g_{K_{M}}-2=-2 \Phi(M)+(q-2) \frac{\Phi(M)}{q-1}+\sum_{i=1}^{r} s_{i} \frac{\Phi(M)}{\Phi\left(P_{i}^{n_{i}}\right)} d_{i} .
$$

As for $g_{K_{M}^{+}}$, if $r=1$, then

$$
2 g_{K_{M}^{+}}-2=(d n-2) \frac{\Phi(M)}{q-1}-d \frac{q^{d(n-1)}-1}{q-1}-d
$$

otherwise,

$$
2 g_{K_{M}^{+}}-2=\frac{1}{q-1}\left(2 g_{K_{M}}-2-(q-2) \frac{\Phi(M)}{q-1}\right)
$$

To provide a relatively elementary proof of the Brumer-Stark conjecture in the function field context involving no algebraic geometry beyond the Riemann-Roch theorem for curves, Hayes [4] defined, in 1985, a normalizing field $H_{\mathfrak{e}}^{*}$ associated with a fixed sgn-normalized Drinfeld module and its extension field $K_{\mathfrak{m}}$, which is an analogue of the classical cyclotomic function field over the rational function field. The purpose of this note is to present explicitly the formulae for the genus of $H_{\mathfrak{e}}^{*}$ and $K_{\mathfrak{m}}$, which were obtained by Hayes [4], and the so-called maximal real subfield $H_{\mathfrak{a}}$ of $K_{\mathfrak{a}}$.

2. Preliminaries. Let $K / \mathbb{F}_{q}$ be a global function field over finite constant field $\mathbb{F}_{q}$ with $q$ elements, and $\infty$ be a fixed prime divisor of $K$ with degree $d_{\infty}$. Let $\mathcal{O}_{K}$ denote the ring of elements of $K$ regular outside of $\infty$, and let $K_{\infty}$ be the completion of $K$ at $\infty$. It is well known ([6], chapter 14) that $\mathcal{O}_{K}$ is a Dedekind ring with a group of units $\mathcal{O}_{K}^{*}=\mathbb{F}_{q}^{*}$ and with class number $h\left(\mathcal{O}_{K}\right)=d_{\infty} h_{K}$, where $h_{K}$ is the class number of $K$. Denote by $\bar{K}_{\infty}$ the algebraic closure of $K_{\infty}$, and $\mathbf{C}_{\infty}$ the completion of $K_{\infty}$ with respect to $\operatorname{ord}_{\infty}(*)$, which is the ord function associated with the prime $\infty$. Denote by $\infty$ also the unique extension of the above fixed prime to $\bar{K}_{\infty}$ and $\mathbf{C}_{\infty}$. It is also well known ([6], chapter 13) that $\mathbf{C}_{\infty}$ is complete and algebraically closed, and will play the role of the complex numbers $\mathbb{C}$ in our context. 
We have known that global function fields are analogues of number fields. In 1981, Hayes [3] invented the normalization theory of the Drinfeld $\mathcal{O}_{K}$-module when $d_{\infty}=1$, to investigate the analytic class number formulae for global function fields. Afterwards, he defined elliptic units in function fields context by this theory, and then computed the index of group of elliptic units in the full unit group. Inspired by this theory, Hayes [4] extended the above normalization theory to the general case, i.e. $d_{\infty}>1$. With the help of this beautiful theory, he provided an elegant and elementary proof of the famous Brumer-Stark conjecture for global function fields. In this note, we mainly study the different divisors and genus of the fields obtained by Hayes [4], i.e. $H_{\mathfrak{e}}^{*}$ and $K_{\mathfrak{m}}$ in Section 4 of [4]. Before starting our topic, we shall first recall some terminologies from [4].

Let $\rho$ be an arbitrary Drinfeld $\mathcal{O}_{K}$-module over $\mathbf{C}_{\infty}$ such that the constant of $\rho_{x}(t)$ is equal to $x$ for any $x \in \mathcal{O}_{K}$. Let $T$ be a subfield of $\mathbf{C}_{\infty}$ containing $K$. We say that $T$ is a field of definition for $\rho$ if $\rho$ is isomorphic over $\mathbf{C}_{\infty}$ to a Drinfeld $\mathcal{O}_{K}$-module $\rho^{\prime}$ such that $\rho_{x}^{\prime}$ has coefficients belonging to $T$ for every $x \in \mathcal{O}_{K}$. For each nonconstant element $x \in \mathcal{O}_{K}$, let $I_{x}(\rho)$ denote the field of invariant of $\rho$ at $x$ (see Definition 6.3 in [2]). Proposition 6.4 and Theorem 6.5 of [2] showed that $I_{x}(\rho)$ is a field of definition for $\rho$ and depends only on the isomorphism class of $\rho$. Thus, $I_{x}(\rho)$ depends only on $\rho$ and not on the choice of $x$. We write $I(\rho)=I_{x}(\rho)$ for any nonconstant $x \in \mathcal{O}_{K}$ and call it the field of invariants of $\rho$. It is easy to see that $I(\rho)$ is the smallest field of definition for $\rho$. Let sgn be a fixed sign function, and $\rho$ be a sgn-normalized Drinfeld $\mathcal{O}_{K}$-module over $\mathbf{C}_{\infty}$ of generic characteristic (note here that $\mathcal{O}_{K} \subset \mathbf{C}_{\infty}$ ). Denote by $H_{1}$ the Hilbert class field of $K$ associated with $\mathcal{O}_{K}$. We know from Hayes [2] that $H_{1}$ is the field of invariants of $\rho^{\mathfrak{a}}$ for any fractional ideal $\mathfrak{a}$ of $\mathcal{O}_{K}$, where $\rho^{\mathfrak{a}}$ is the Drinfeld module corresponding to the lattice $\mathfrak{a}$. By Corollary 8.12 of [2], it is unramified of degree $h\left(\mathcal{O}_{K}\right)$ over $K$, and its constant field has degree $d_{\infty}$ over $\mathbb{F}_{q}$. Thus, if $d_{\infty}=1$, then $H_{1} / K$ is a geometric extension, and we can get the genus of $H_{1}$ as follows by the Riemann-Hurwitz formula:

$$
g_{H_{1}}=1+h_{K}\left(g_{K}-1\right)
$$

With the help of Corollary 4.8 of [4], if $d_{\infty}>1$, then a Drinfeld $\mathcal{O}_{K}$-module of generic characteristic over $H_{1}$ cannot be a sgn-normalized Drinfeld module. Assume that $d_{\infty}=1$ and $\rho$ is a sgn-normalized Drinfeld $\mathcal{O}_{K}$-module over $H_{1}$ of generic characteristic hereafter. Let $I^{*}(\rho)$ be the subfield of $\mathbf{C}_{\infty}$ generated by the coefficients of polynomial $\rho_{x}, x \in \mathcal{O}_{K}-\{0\}$. Hayes pointed out [2] that $I^{*}(\rho)$ is independent of the choice of $\rho$ up to isomorphism. Denote by $H_{\mathfrak{e}}^{*}$ the common field $I^{*}(\rho)$ for the sgnnormalized Drinfeld $\mathcal{O}_{K}$-module of generic characteristic. When $d_{\infty}=1$, we can claim from Theorem 4.10 of [4] that the normalizing field of $\rho$, denoting by $H_{\mathfrak{e}}^{*}$, is exactly $H_{1}$. It can be inferred by the same theorem that $H_{1}\left(\Lambda_{\mathfrak{a}}\right) / K$ is an abelian extension for any nonzero ideal $\mathfrak{a} \subset \mathcal{O}_{K}$, where $\Lambda_{\mathfrak{a}}$ is the set of $\mathfrak{a}$-torsion points of $\rho$. Let $G_{\infty}$ be the decomposition group of $\infty$ in $H_{1}\left(\Lambda_{\mathfrak{a}}\right)$. Then, the fixed field of $G_{\infty}$ in $H_{1}\left(\Lambda_{\mathfrak{a}}\right)$ is the ray class field $H_{\mathfrak{a}}$ of $\mathcal{O}_{K}$ associated with $\mathfrak{a}$. For any prime of $H_{\mathfrak{a}}$ lying over $\infty$, it is totally ramified in $H_{1}\left(\Lambda_{\mathfrak{a}}\right)$ with the ramification index equal to $q-1$. By these facts, we can easily prove the following simple facts:

FACT 2.1. The two function field extensions $H_{\mathfrak{a}} / K$ and $H_{1}\left(\Lambda_{\mathfrak{a}}\right) / K$ are both geometric extensions. 
Proof. Denote by $\mathfrak{p}_{\infty}$ and $\mathfrak{P}_{\infty}$ respectively primes of $H_{\mathfrak{a}}$ and $H_{1}\left(\Lambda_{\mathfrak{a}}\right)$ lying over $\infty$, and $F$ and $E$ the constant fields of $H_{\mathfrak{a}}$ and $H_{1}\left(\Lambda_{\mathfrak{a}}\right)$, respectively. By the proof of Proposition 7.7 in [6], we obtain

$$
\left[F: \mathbb{F}_{q}\right] \operatorname{deg}_{H_{\mathfrak{a}}} \mathfrak{p}_{\infty}=f\left(\mathfrak{p}_{\infty} / \infty\right) d_{\infty}
$$

and

$$
[E: F] \operatorname{deg}_{H_{1}\left(\Lambda_{\mathfrak{a}}\right)} \mathfrak{P}_{\infty}=f\left(\mathfrak{P}_{\infty} / \mathfrak{p}_{\infty}\right) \operatorname{deg}_{H_{\mathfrak{a}}} \mathfrak{p}_{\infty},
$$

where $f\left(\mathfrak{p}_{\infty} / \infty\right)$ and $f\left(\mathfrak{P}_{\infty} / \mathfrak{p}_{\infty}\right)$ are residue class degrees. We note that $\infty$ splits completely in $H_{\mathfrak{a}} / K$ and $\mathfrak{p}_{\infty}$ is totally ramified in $H_{1}\left(\Lambda_{\mathfrak{a}}\right) / H_{\mathfrak{a}}$. Therefore, $f\left(\mathfrak{p}_{\infty} / \infty\right)=$ $f\left(\mathfrak{P}_{\infty} / \mathfrak{p}_{\infty}\right)=1$. Combining this equality with the fact $d_{\infty}=1$, we can claim that $\left[F: \mathbb{F}_{q}\right]=\operatorname{deg}_{H_{\mathfrak{a}}} \mathfrak{p}_{\infty}=1$, which in turn means that $[E: F]=1$. Thus, $E=F=\mathbb{F}_{q}$, and our proof is complete.

3. Main results. With comments of the previous section, we can now discuss the genus of $H_{1}\left(\Lambda_{\mathfrak{a}}\right)$ and $H_{\mathfrak{a}}$. To ease the notation, we denote by $K_{\mathfrak{a}}$ the field $H_{1}\left(\Lambda_{\mathfrak{a}}\right)$. It is worth noting that if $\mathfrak{p}^{e}$ is the exact power of $\mathfrak{p}$ dividing $\mathfrak{a}$, then any prime $\mathcal{P}$ of $H_{1}$ lying above $\mathfrak{p}$ is ramified in $K_{\mathfrak{a}} / H_{1}$ with the ramification index $\Phi_{\mathcal{O}_{K}}\left(\mathfrak{p}^{e}\right)$, where $\Phi_{\mathcal{O}_{K}}\left(\mathfrak{p}^{e}\right)$ denotes the order of multiplicative group $\left(\mathcal{O}_{K} / \mathfrak{p}^{e}\right)^{*}$; if $\mathfrak{p} \nmid \mathfrak{a}$, then $\mathcal{P}$ dose not ramify in $K_{\mathfrak{a}} / H_{1}$.

First, we consider the case that $\mathfrak{a}=\mathfrak{p}^{n}$ with $n \geq 1$. The following theorem will give the different divisor of the extension $K_{\mathfrak{a}} / K$.

THEOREM 3.1. Let $\mathfrak{a}=\mathfrak{p}^{n}$ with $n \geq 1$ for prime ideal $\mathfrak{p}$. The different divisor of the extension $K_{\mathfrak{a}} / K$ is

$$
D_{K \mathfrak{a} / K}=s \sum_{\mathcal{P} \mid \mathfrak{p}} \mathfrak{P}+(q-2) \sum_{\mathfrak{P}_{\infty} \mid \infty} \mathfrak{P}_{\infty},
$$

where $\mathfrak{P}$ is the unique prime of $K_{\mathfrak{a}}$ lying above $\mathcal{P}$, which is a prime of $H_{1}$ lying over $\mathfrak{p}$, and $s=n \Phi_{\mathcal{O}_{K}}(\mathfrak{a})-\frac{\Phi_{\mathcal{O}_{K}}(\mathfrak{a})}{\Phi_{\mathcal{O}_{K}}(\mathfrak{p})}$.

Proof. Note that the proof of our conclusion is similar to that of Hayes Theorem 4.1 [1]. We state it here for completeness.

We note first that only the primes $\mathfrak{p}$ and $\infty$ are ramified in $K_{\mathfrak{a}}$. Since $\infty$ splits completely in $H_{\mathfrak{a}}$ and each prime of $H_{\mathfrak{a}}$ lying above $\infty$ is totally ramified in $K_{\mathfrak{a}}$, the ramification index of $\infty$ in $K_{\mathfrak{a}}$ is $q-1$, and thus the $\infty$-part of $D_{K_{\mathfrak{a}} / K}$ is $(q-2)$ $\sum_{\mathfrak{P}_{\infty} \mid \infty} \mathfrak{P}_{\infty}$. So we only have to show the value of $s$. To determine $s$, we need to compute the exponent of the different divisor $D_{K_{\mathfrak{a}} / H_{1}}$ at the prime above $\mathcal{P}$ by local considerations, where $\mathcal{P}$ is the prime of $H_{1}$ lying over $\mathfrak{p}$. Let $K_{\mathfrak{a}, \mathfrak{P}}$ and $H_{1, \mathcal{P}}$ denote the completion of $K_{\mathfrak{a}}$ and $H_{1}$ at primes $\mathfrak{P}$ and $\mathcal{P}$, respectively. Then, $K_{\mathfrak{a}, \mathfrak{P}} / H_{1, \mathcal{P}}$ is a totally ramified extension of local fields, and $K_{\mathfrak{a}, \mathfrak{P}}$ can be generated by a single root $\lambda \in \Lambda_{\mathfrak{a}}$ of $f(u)=\frac{\rho_{\mathrm{a}}(u)}{\rho_{\mathrm{p} n-1}(u)}$. By Proposition 7.6 in [2], we get that $f(u)$ is an Eisenstein polynomial at $\mathcal{P}$, and this means that $\lambda^{i}$ with $0 \leq i<\Phi_{\mathcal{O}_{K}}\left(\mathfrak{p}^{n}\right)$ constitute an integral basis for this local extension. By the theory of local fields, we claim that the discriminant of this extension is generated by the norm of $f^{\prime}(\lambda)$. Note that $\rho_{\mathfrak{a}}(u)=\rho_{\mathfrak{p}^{n-1}}(u) f(u)$, where $\rho_{\mathfrak{a}}$ is the unique isogeny from $\rho$ to $\mathfrak{a} * \rho$ up to isomorphism (for details see [2]). By differentiating both sides of the above equality and then replacing $u$ by $\lambda$, we can get that 
$D\left(\rho_{\mathfrak{a}}\right)=\rho_{\mathfrak{p}^{n-1}}(\lambda) f^{\prime}(\lambda)$, where $D\left(\rho_{\mathfrak{a}}\right)$ denotes the constant of $\rho_{\mathfrak{a}}(u)$. Since $\rho_{\mathfrak{p}^{n-1}}(\lambda) \in \Lambda_{\mathfrak{p}}$ and the valuation at $\mathcal{P}$ of nonzero elements of $\Lambda_{\mathfrak{p}}$ is one, and the valuation of $D\left(\rho_{\mathfrak{a}}\right)$ at $\mathcal{P}$ is $n \Phi_{\mathcal{O}_{K}}(\mathfrak{a})$, we obtain that the valuation at $\mathcal{P}$ of the norm of $f^{\prime}(\lambda)$ is $n \Phi_{\mathcal{O}_{K}}(\mathfrak{a})-$ $\frac{\Phi_{\mathcal{O}_{K}}(\mathfrak{a})}{\Phi_{\mathcal{O}_{K}}(\mathfrak{p})}$. By returning to the global circumstance again, we can finally establish our conclusion.

With the aid of the above theorem, we can deal with the general case by means of the transitive property of different divisors.

COROLlaRY 3.2. Let $\mathfrak{a}=\mathfrak{p}_{1}^{n_{1}} \cdots \mathfrak{p}_{r}^{n_{r}}$ with $r \geq 2$ and $n_{i}>0,1 \leq i \leq r$. The different divisor of $K_{\mathfrak{a}} / K$ is

$$
D_{K_{\mathfrak{a}} / K}=\sum_{i=1}^{r} s_{i}\left(\sum_{\mathcal{P}_{i} \mid \mathfrak{P}_{i}} \sum_{\mathfrak{P}_{i} \mid \mathcal{P}_{i}} \mathfrak{P}_{i}\right)+(q-2) \sum_{\mathfrak{P}_{\infty} \mid \infty} \mathfrak{P}_{\infty},
$$

where $s_{i}=n_{i} \Phi_{\mathcal{O}_{K}}\left(\mathfrak{p}_{i}^{n_{i}}\right)-\frac{\Phi_{\mathcal{O}_{K}}\left(\mathfrak{p}_{i}^{n_{i}}\right)}{\Phi_{\mathcal{O}_{K}}\left(\mathfrak{p}_{i}\right)}$.

Proof. Since the ramification index of $\infty$ in $K_{\mathfrak{a}}$ is also $q-1$, the coefficients of prime $\mathfrak{P}_{\infty} \mid \infty$ are all $q-2$, and thus the $\infty$-part of the different divisor can be attained. For the finite parts, we only have to consider the case $r=2$, and the general case can be done by the same arguments. Since only primes of $H_{1}$ lying above $\mathfrak{p}_{1}$ (resp. $\mathfrak{p}_{2}$ ) are ramified in $K_{\mathfrak{p}_{1}{ }_{1}} / H_{1}\left(\right.$ resp. $\left.K_{\mathfrak{p}_{2}{ }_{2}} / H_{1}\right), K_{\mathfrak{p}_{1}{ }_{1}}$ and $K_{\mathfrak{p}_{2}{ }_{2}}$ are linearly disjoint over $H_{1}$, the finite parts $\left(D_{K_{\mathbf{p}_{i}} / K}\right)_{0}, i=1,2$ are relatively prime. By the transitive property of the different divisor, we get that

$$
\left(D_{K_{\mathfrak{a}} / K_{\mathfrak{p}_{1}}{ }_{1}}\right)_{0}+i_{K_{\mathfrak{a}} / K_{\mathfrak{p}_{1}} n_{1}}\left(D_{K_{\mathfrak{p}_{1}} n_{1} / K}\right)_{0}=\left(D_{K_{\mathfrak{a}} / K_{\mathfrak{p}_{2}}}\right)_{0}+i_{K_{\mathfrak{a}} / K_{\mathfrak{p}_{2}}}\left(D_{K_{\mathbf{p}_{2}} / n_{2}}\right)_{0},
$$

where $i_{K_{\mathfrak{a}} / K_{\mathfrak{p}_{i}^{i}}{ }_{i}}$ is the extension of the divisors map. With the aid of the above statements, we claim that, for $i \neq j$,

$$
\left(D_{K_{\mathfrak{a}} / K_{\mathfrak{p}_{i}^{n_{i}}}}\right)_{0}=i_{K_{\mathfrak{a}} / K_{\mathfrak{p}_{j}{ }_{j}}}\left(D_{\left.K_{\mathfrak{p}_{j} n_{j} / K}\right)_{0}}\right.
$$

Thus, the expression of finite parts of the different divisor can be obtained by above theorem. By combining this with the above $\infty$-part, we complete our proof.

In order to continue our topic, we need the following lemma, which is interesting in itself.

LEMMA 3.3. Let $\mathfrak{a}=\mathfrak{p}_{1}^{n_{1}} \cdots \mathfrak{p}_{r}^{n_{r}}$ with $r \geq 2$ and $n_{i}>0,1 \leq i \leq r$, and $\mathcal{O}_{\mathfrak{a}}$ be the integral closure of $\mathcal{O}_{K}$ in $K_{\mathfrak{a}}$. Then, a generator of $\Lambda_{\mathfrak{a}}$ is a unit in $\mathcal{O}_{\mathfrak{a}}$.

Proof. First, it is easy to show that $\Lambda_{\mathfrak{a}} \subseteq \mathcal{O}_{\mathfrak{a}}$ by the properties of a sgn-normalized Drinfeld module (for details see [4]). We known that $\Lambda_{\mathfrak{a}}$ is isomorphic to $\mathcal{O}_{K} / \mathfrak{a}$ as $\mathcal{O}_{K}$-modules. Suppose that $\lambda \in \Lambda_{\mathfrak{a}}$ be a generator of $\Lambda_{\mathfrak{a}}$. In the first place, we prove the case $r=2$. Afterwards, we can prove the general case by induction. Assume now that $\mathfrak{a}=\mathfrak{p}_{1}^{n_{1}} \mathfrak{p}_{2}^{n_{2}}$. Set $\lambda_{1}=\rho_{\mathfrak{a} / \mathfrak{p}_{1}{ }^{n_{1}}}(\lambda)$ and $\lambda_{2}=\rho_{\mathfrak{a} / \mathfrak{p}_{2}{ }^{n_{2}}}(\lambda)$. Then, $\lambda_{1}$ and $\lambda_{2}$ are the generators of $\Lambda_{\mathfrak{p}_{1}^{n_{1}}}$ and $\Lambda_{\mathfrak{p}_{2} n_{2}}$, respectively. By Lemma 4.19 of [4], they generate $\mathfrak{p}_{i}^{n_{i}} \mathcal{O}_{\mathfrak{p}_{i}^{n_{i}}}$ in the subfields $K_{\mathfrak{p}_{i}^{n_{i}}}$ of $K_{\mathfrak{a}}$ for $i=1$, 2, where $\mathcal{O}_{\mathfrak{p}_{i}^{n_{i}}}$ is the integral closure of $\mathcal{O}_{K}$ in $K_{\mathfrak{p}_{i}^{n_{i}}}$. Since these two generated ideals are relatively prime in $\mathcal{O}_{\mathfrak{a}}$, there are elements $\alpha$ and $\beta$ in $\mathcal{O}_{\mathfrak{a}}$ 
such that $\alpha \lambda_{1}+\beta \lambda_{2}=1$. Note here that $\lambda$ divides both $\lambda_{1}$ and $\lambda_{2}$, then it divides units, and thus $\lambda$ is a unit.

REMARK 3.4. The result of the above lemma can be considered as the generalization of Proposition 12.6 of [6].

With the above preparation, we can now compute the different divisor of the extension $K_{\mathfrak{a}} / H_{\mathfrak{a}}$.

THEOREM 3.5. Let $\mathfrak{a}=\mathfrak{p}_{1}^{n_{1}} \cdots \mathfrak{p}_{r}^{n_{r}}$ be a nonzero ideal of $\mathcal{O}_{K}$ with $r \geq 1$ and $n_{i}>0$, $1 \leq i \leq r$. If $r=1$, then

$$
D_{K_{\mathfrak{a}} / H_{\mathfrak{a}}}=(q-2)\left(\sum_{\mathfrak{P}_{1} \mid \mathfrak{p}_{1}} \mathfrak{P}_{1}+\sum_{\mathfrak{P}_{\infty} \mid \infty} \mathfrak{P}_{\infty}\right)
$$

otherwise,

$$
D_{K_{\mathfrak{a}} / H_{\mathfrak{a}}}=(q-2) \sum_{\mathfrak{P}_{\infty} \mid \infty} \mathfrak{P}_{\infty}
$$

Proof. Because $H_{\mathfrak{a}}$ is the decomposition field of $\infty$ in $K_{\mathfrak{a}}$, and primes of $K_{\mathfrak{a}}$ lying above $\infty$ are totally ramified over $H_{\mathfrak{a}}$, the $\infty$-part of the different divisor is always equal to $(q-2) \sum_{\mathfrak{P}_{\infty} \mid \infty} \mathfrak{P}_{\infty}$ in any case. First, we consider the case $r=1$. We note that only primes of $H_{\mathfrak{a}}$ lying above $\mathfrak{p}_{1}$ are totally ramified in $K_{\mathfrak{a}}$ with the ramification index $q-1$, and thus the coefficients of primes appearing in different are all $q-2$. Now we deal with another case, i.e. $r>1$. Actually, we will show that $K_{\mathfrak{a}} / H_{\mathfrak{a}}$ is unramified except at the primes lying over $\infty$. Note that $K_{\mathfrak{a}}=H_{1}(\lambda)$ and $H_{\mathfrak{a}}=H_{1}\left(\lambda^{q-1}\right)$ for a generator of $\lambda \in \Lambda_{\mathfrak{a}}$. The minimal irreducible polynomial of $\lambda$ over $H_{\mathfrak{a}}$ is $f(x)=x^{q-1}-\lambda^{q-1}$. Thus, the discriminant of $\lambda$ is $\left(\lambda^{(q-1)(q-2)}\right)$. In the theory of global fields, the discriminant of $K_{\mathfrak{a}} / H_{\mathfrak{a}}$ divides the ideal generated by the discriminant of $\lambda$. Using Lemma 3.3, we have that any finite prime of $H_{\mathfrak{a}}$ is unramified in $K_{\mathfrak{a}}$, and this establishes our theorem.

Having obtained explicit formulae for different divisors, we can compute the genus of these function fields.

COROLlary 3.6. With notation as Corollary 3.2, set $d_{i}=\operatorname{deg}_{K} \mathfrak{p}_{i}$ for $1 \leq i \leq r$. Then we get that if $r=1$, then

$$
2 g_{K_{\mathfrak{a}}}-2=h_{K} \Phi_{\mathcal{O}_{K}}(\mathfrak{a})\left(2 g_{K}-2\right)+h_{K} s_{1} d_{1}+\frac{h_{K} \Phi_{\mathcal{O}_{K}}(\mathfrak{a})(q-2)}{q-1}
$$

otherwise,

$$
2 g_{K_{\mathfrak{a}}}-2=h_{K} \Phi_{\mathcal{O}_{K}}(\mathfrak{a})\left(2 g_{K}-2\right)+h_{K} \Phi_{\mathcal{O}_{K}}(\mathfrak{a}) \sum_{i=1}^{r} \frac{s_{i} d_{i}}{\Phi_{\mathcal{O}_{K}}\left(\mathfrak{p}_{i}^{n_{i}}\right)}+\frac{h_{K} \Phi_{\mathcal{O}_{K}}(\mathfrak{a})(q-2)}{q-1},
$$

where $h_{k}$ is the class number of $K$.

Proof. Note here that $K_{\mathfrak{a}} / K$ is a geometric extension. With the help of Theorem 3.1 and Corollary 3.2, we can compute the genus of $K_{\mathfrak{a}}$ by the Riemann-Hurwitz formula. 
COROLlary 3.7. With the notation as Theorem 3.5, if $r=1$, then

$$
2 g_{H_{\mathfrak{a}}}-2=\frac{h_{K} \Phi_{\mathcal{O}_{K}}(\mathfrak{a})\left(2 g_{K}-2\right)}{q-1}+\frac{h_{K} d_{1}\left(s_{1}+2-q\right)}{q-1} ;
$$

otherwise,

$$
2 g_{H_{\mathfrak{a}}}-2=\frac{h_{K} \Phi_{\mathcal{O}_{K}}(\mathfrak{a})\left(2 g_{K}-2\right)}{q-1}+\frac{h_{K} \Phi_{\mathcal{O}_{K}}(\mathfrak{a})}{q-1} \sum_{i=1}^{r} \frac{s_{i} d_{i}}{\Phi_{\mathcal{O}_{K}}\left(\mathfrak{p}_{i}^{n_{i}}\right)}
$$

We give some remarks about above formulae to conclude our note.

REMARK 3.8. Using sgn-normalized Drinfeld modules over $H_{1}$ of generic characteristic, we get explicit formulae for different divisors of $K_{\mathfrak{a}} / K$ and $K_{\mathfrak{a}} / H_{\mathfrak{a}}$, and the genus of $K_{\mathfrak{a}}$ and $H_{\mathfrak{a}}$. We should note that our methods to determine these formulae are valid only in the case $d_{\infty}=1$. When $d_{\infty}>1$, the normalizing field of any sng-normalized Drinfeld module of generic characteristic is not equal to $H_{1}$. If we assume that $K$ is the rational function field $\mathbb{F}_{q}(T)$ and $\infty=\frac{1}{T}$, then our above formulae are in accordance with Hayes's [1] Theorem 4.1 and Kida and Murabayashi's [5] Corollary 1, and thus these formulae can be regarded as the generalizations of the results obtained by Hayes and Kida.

Acknowledgements. We thank our adviser Professor Qin Hourong for many helpful suggestions. We also thank the referees for careful reading of the manuscript and the suggestions on the writing of the paper. This work was supported by the NSFC (Nos. 11171141, 11071110 and 10971091), the SRFDP (No. 200802840003) and the NSFJ (Nos. BK2010007 and BK2010362).

\section{REFERENCES}

1. D. Hayes, Explicit class field theory for rational function fields, Trans. Amer. Math. Soc. 189 (1974), 77-91.

2. D. Hayes, Explicit class field theory in global function fields, Studies in Algebra and Number Theory, Adv. Math. Supplementary Studies 6 (1979), 173-217.

3. D. Hayes, Analytic class number formulas in global function fields, Invent. Math. $\mathbf{6 5}$ (1981), 49-69.

4. D. Hayes, Stickelberger elements in function fields, Compos. Math. 55 (1985), 209-239.

5. M. Kida and N. Murabayashi, Cyclotomic function fields with divisor class number one, Tokyo J. Math. 14(1) (1991), 45-56.

6. M. Rosen, Number theory in function fields, Graduate Texts in Mathematics, vol. 210 (Springer, Berlin, 2002). 